\title{
Dientes Fósiles de Carcharocles megalodon: La Colección del Museo Universitario de Chieti (Italia). Parte I: Análisis Morfométrico
}

\author{
Fossil teeth of Carcharocles megalodon: The Collection of the \\ University Museum of Chieti (Italy). Part I: Morphometric Analysis
}

Joan Viciano'; Sandra López-Lázaro² \& Ruggero D’Anastasio ${ }^{1}$

VICIANO, J.; LÓPEZ-LÁZARO, S. \& D'ANASTASIO, R. Dientes fósiles de Carcharocles megalodon: La colección del Museo Universitario de Chieti (Italia). Parte I: Análisis Morfométrico. Int. J. Morphol., 36(4):1378-1385, 2018.

RESUMEN: La especie extinta de tiburón Carcharocles megalodon (Clase: Chondrichthyes; Orden: Lamniformes) es considerado uno de los depredadores más grandes y más poderosos que ha poblado los mares templados de todo el mundo entre el Mioceno medio y el Plioceno (15,9-2,6 Ma). La reconstrucción de su historia evolutiva y ecología se basa principalmente en el estudio morfológico y métrico de los dientes, que representan la abrumadora mayoría de los restos fósiles de esta especie con esqueleto cartilaginoso hallados hasta hoy. En el presente artículo presentamos las características morfológicas y métricas de los dientes de Carcharocles megalodon que se conservan en el Museo Universitario de Chieti (Italia). La colección se compone de nueve dientes de la arcada superior, que pertenecerían a diferentes ejemplares de tamaño variable entre los 6,27-14,50 m y los 2.510-35.918 kg. de peso. Los dientes implementan el registro fósil existente de Carcharocles megalodon, de modo que los datos morfométricos recabados contribuirán a mejorar el conocimiento de los estudios filogenéticos y ecológicos de esta especie.

PALABRAS CLAVE: Megalodonte; Diente fósil; Tiburón extinto; Lamniformes; Tamaño; Masa corporal.

\section{INTRODUCCIÓN}

El megalodonte ("diente grande"; derivado de los términos griegos $\mu \varepsilon \gamma \alpha \varsigma$ - mega, "grande"—, y odovs — odon, "diente"-), es considerado uno de los depredadores más grandes y más poderosos jamás existido en la historia de los vertebrados. Corresponde a una especie extinta de tiburón que vivió aproximadamente desde el Mioceno medio (hace aproximadamente 16 millones de años) hasta el final del Plioceno (hace aproximadamente 2,6 millones de años) (Gottfried et al., 1996; Purdy, 1996; Pimiento et al., 2010). Sus dimensiones, estimadas a partir del tamaño de los dientes, podrían superar los $16 \mathrm{~m}$. de longitud, más del doble de la longitud máxima del gran tiburón blanco con sus 6,4 m. (Bigelow \& Schroeder, 1948; Randall, 1973; Compagno, 1984).

Clasificación taxonómica. La clasificación taxonómica del megadolonte ha sido objeto de debate científico por cerca de un siglo y a día de hoy la controversia aún persiste con dos interpretaciones principales bien diferenciadas. Se clasifica bien como Carcharodon megalodon (bajo la familia Lamnidae), o bien como Carcharocles megalodon (bajo la familia Otodontidae) (Pimiento et al., 2010). La visión tradicional es que el megalodonte debería ser clasificado dentro del género Carcharodon junto con el gran tiburón blanco actual (Carcharodon carcharias), argumentando una gran similitud tanto en morfología como en la estructura de los dientes. Los autores que apoyan esta filogenia se basan en ciertas características que comparten ambas especies como, por ejemplo: (i) la presencia de una gradación ontogénica mediante la cual el aserramiento tosco presente en los dientes de los ejemplares jóvenes del gran tiburón blanco pasa a ser más fino en los ejemplares adultos, siendo estos últimos de gran semejanza a los del megalodonte; (ii) la similitud morfológica de los dientes en los ejemplares jóvenes de ambas especies; (iii) la presencia de un segundo diente anterior simétrico; o (iv) grandes dientes intermedios que se inclinan medialmente, entre otras características (Uyeno et al., 1989; Applegate, 1991; Gottfried et al., 1992, 1996; Applegate \& Espinosa-Arrubarrena, 1996; Purdy, 1996). Sin embargo, un número creciente de investigadores ha cuestionado la relación filogenética entre $C$. megalodon y $C$. carcharias y ha propuesto el nuevo género Carcharocles 
para clasificar el tiburón megalodonte, argumentando la evolución convergente como la razón principal para la similitud de los dientes de ambas especies (Casier, 1960; Glickman, 1964; Capetta, 1987; Ward \& Bonavia, 2001; Nyberg et al., 2006; Ehret et al., 2009, 2012; Pimiento et al., 2010). A pesar que los argumentos que apoyan al género Carcharocles tienen mayor número de apoyos en la comunidad científica, la clasificación taxonómica tradicional aún tiene amplia aceptación y uso en la literatura. No obstante, ambos géneros se incluyen en el Orden Lamniformes y el actual gran tiburón blanco (C. carcharias) sigue siendo la especie morfológicamente más cercana a $C$. megalodon, de modo que el aspecto y las dimensiones de este último se reconstruyen precisamente a partir de esta similitud. En el presente artículo, nosotros clasificaremos el megalodonte dentro del género Carcharocles, siguiendo la taxonomía presente en la literatura científica más reciente (p. ej.: Pimiento et al., 2014; 2015).

Los dientes fósiles de $\boldsymbol{C}$. megalodon del Museo Universitario de Chieti (Italia). La historia evolutiva y la paleoecología de $C$. megalodon se basa principalmente en el análisis de los dientes y de algunos centros vertebrales, los cuales representan los únicos restos fósiles hallados en los sedimentos mio-pliocénicos a causa de la naturaleza cartilaginosa de su esqueleto (Reolid \& Molina, 2015). El ejemplar más completo de megalodonte consiste en aproximadamente 150 centros vertebrales hallados en el yacimiento paleontológico de Antwerp (Bélgica, Mioceno) (Leriche, 1926).

Son numerosos los yacimientos paleontológicos que han proporcionado dientes fósiles de $C$. megalodon, estando distribuidos tales yacimientos por casi todo el mundo (Uyeno et al., 1989; Purdy et al., 2001; Pimiento et al., 2015; Reolid \& Molina, 2015). No obstante, son solo algunos centenares los dientes en buen estado de conservación útiles para estudios morfológicos y métricos. Además, estos especímenes se encuentran distribuidos en modo disperso por Museos y Universidades de varios continentes, a menudo conservados en ubicaciones muy distantes unos de otros. Por ejemplo, Pimiento et al. (2014) visitaron más de 18 localidades en Europa, Australia, América del Norte y del Sur para recopilar datos sobre poco más de 500 dientes fósiles. Si consideramos el hecho que los dientes se diferencian por tipología y datación, parece oportuno y necesario poner a disposición de los investigadores la mayor parte de los especímenes, incluso aquellos conservados y almacenados en Museos y Universidades que, lamentablemente, aún no se encuentran accesibles; el fin último es obtener un mayor número de datos y poder proceder con estudios más complejos como, por ejemplo, aquellos relacionados con la variabilidad geográfica, sincrónica y diacrónica de la especie.
El Museo Universitario de la Universidad "G. d'Annunzio" de Chieti-Pescara (Italia), dedicado al conocimiento y divulgación de la historia de las ciencias biomédicas, posee una vasta colección de dientes compuesta principalmente por dientes humanos, sobre los que se han llevado a cabo numerosos estudios antropológicos y paleopatológicos (p. ej.: D'Anastasio et al., 2013; Viciano et al., 2012, 2015a, 2015b), pero también incluye dientes de otras especies animales, entre los que se encuentran nueve especímenes de $C$. megalodon. Los dientes de megalodonte se presentan en buen estado de conservación, de modo que su descripción y puesta en conocimiento a la comunidad científica puede complementar las colecciones ya existentes y contribuir a los estudios sobre las relaciones filogenéticas de C. megalodon con otros tiburones extintos y modernos y su ecología. Los datos sobre la colección dental de $C$. megalodon del Museo Universitario de Chieti se publicarán en dos partes diferenciadas: la primera parte presentará las características morfológicas y métricas de los dientes, que son la base de la reconstrucción de la línea evolutiva de los tiburones megalodontes; la segunda parte ilustrará las patologías que, además de proporcionar información útil para comprender y reconstruir la biología de $C$. megalodon, pueden dilucidar su patocenosis, es decir, el conjunto de enfermedades que afectaron esta especie (Rothschild \& Martin, 1993) Ver D’Anastasio et al. (2018) para mayor detalle del análisis paleopatológico.

\section{MATERIAL Y MÉTODO}

Los nueve dientes de $C$. megalodon incluidos en la colección odontológica del Museo Universitario de Chieti proceden del yacimiento paleontológico de Lee Creek Mine (Carolina del Norte, EE.UU.) (Purdy et al., 2001). Los especímenes mencionados en este artículo están inventariados con los códigos \#118, \#119, \#120, \#165, \#180, \#188, \#190, \#191 y \#192.

Nomenclatura dental. Los dientes han sido identificados y clasificados sobre la base de las indicaciones morfológicas y métricas proporcionadas por los autores que en el pasado han estudiado C. megalodon y C. carcharias (Applegate \& Espinosa-Arrubarrena, 1996; Hubbell, 1996; Mollet et al., 1996), y denominados siguiendo la nomenclatura propuesta por Applegate (1965), posteriormente modificada por Applegate \& Espinosa-Arrubarrena (1996). Se pueden distinguir cuatro clases de dientes en sus mandíbulas, dispuestos en orden desde la línea media: (i) dientes anteriores (A); (ii) dientes intermedios (Int); (iii) dientes laterales (L); y (iv) dientes posteriores (P). Para diferenciar los diferentes tipos de dientes dentro de la clase de dientes anteriores, se 


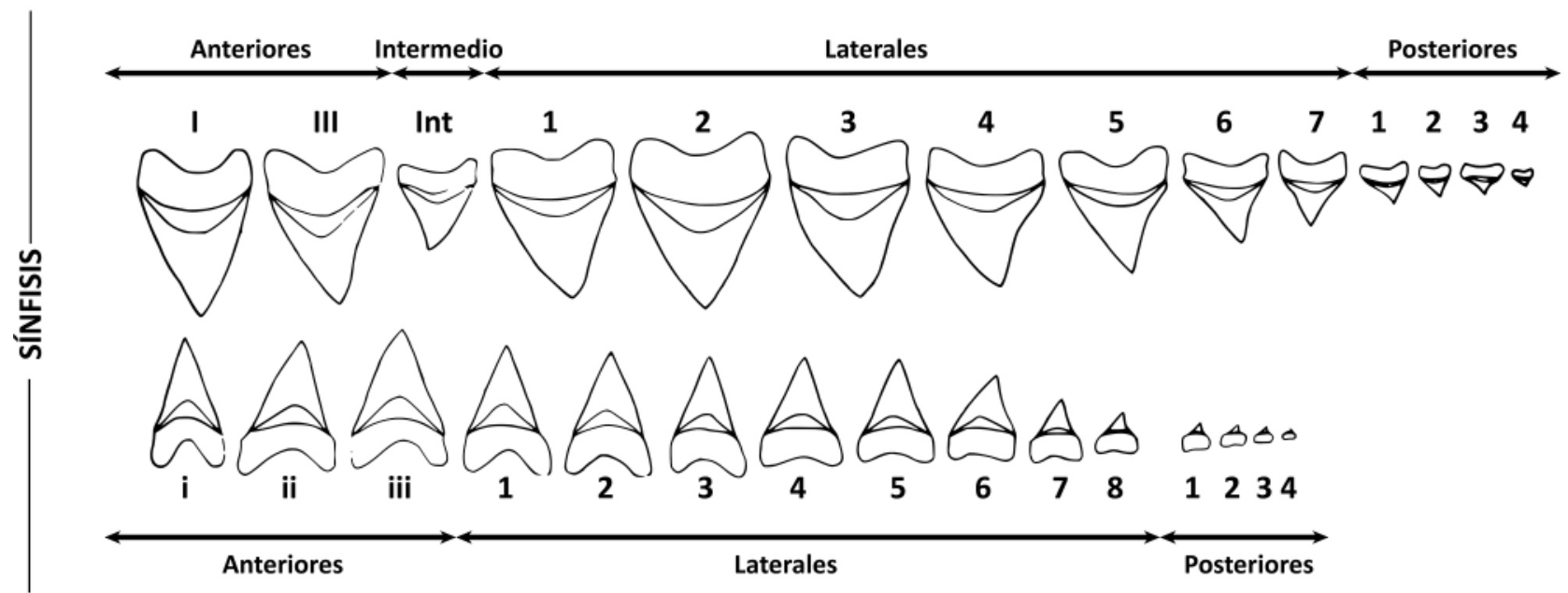

Fig. 1. Fórmula dental de Carcharodon megalodon.

utiliza la numeración romana (en mayúscula para los dientes superiores y en minúscula para los dientes inferiores; p. ej.: 'AI' corresponde al primer diente anterior superior, 'Aiii' corresponde al tercer diente anterior inferior). Respecto a la fórmula dental primitiva de los Lamniformes (basada en el género Carcharodon), que prevé tres dientes anteriores, ya sea superiores que inferiores, $C$. megalodon posee solo dos dientes anteriores superiores y el diente faltante resulta ser el segundo superior (Applegate \& Espinosa-Arrubarrena, 1996). El diente intermedio está presente solo en la arcada superior. En el caso de los dientes laterales, en ambas mandíbulas se numeran los diferentes tipos de dientes dentro de su respectiva clase mediante el uso de números arábigos, comenzando la numeración por el diente más cercano a la sínfisis. Además, se añade la inicial ' $U$ ' o ' $L$ ', dependiendo si el diente es superior o inferior (p. ej.: 'UL3' corresponde al tercer diente lateral superior, 'LL2' corresponde al segundo diente lateral inferior). La Figura 1 muestra la representación de la dentición de C. megalodon en relación a los tipos funcionales de dientes y su disposición dentro de las mandíbulas.

Mediciones dentales. Cada uno de los dientes ha sido descrito siguiendo las mediciones tradicionalmente usadas por los diversos autores (p. ej.: Hubbell, 1996; Reolid \& Molina, 2015) (Fig. 2). En total se han tomado las siguientes 14 medidas: (1) Longitud total del diente (TH; total height): distancia máxima entre la línea tangente a los puntos más inferiores de los lóbulos de la raíz del diente y la línea paralela a esta última, pasando por el ápice de la corona; (2) anchura máxima del diente (TW; total width): distancia máxima entre las líneas paralelas y tangentes a los puntos más externos del diente; (3) diámetro bucolingual (BL; buccolingual diameter): distancia máxima entre las superficies lingual y bucal; (4) altura de la corona ( $\mathrm{CH}$; crown height): distancia vertical entre la línea recta que pasa por las extensiones inferiores del esmalte adyacentes a la raíz en la base de la corona y la línea paralela que pasa por el ápice del diente; (5) anchura de la corona (CW; crown width): parte más ancha del esmalte, en la base de la corona; (6) número de aserraduras del margen mesial (MM; mesial margin): número de aserraduras a lo largo del margen mesial de la corona; (7) número de aserraduras del margen distal (DM; distal margin): número de aserraduras a lo largo del margen distal de la corona; (8) contorno del margen mesial (MC; mesial contour): longitud del margen mesial del diente; (9) contorno del margen distal (DC; distal contour): longitud del margen distal del diente; (10) densidad mesial (MD; mesial density): número de aserraduras por centímetro, a lo largo del margen mesial de la corona; es igual a la proporción MM/ MC; (11) densidad distal (DD; distal density): número de aserraduras por centímetro, a lo largo del margen distal de la corona; es igual a la proporción DM/DC; (12) cuerda mesial (Mc; mesial cord): distancia entre el ápice de la corona y la extensión mesial inferior del esmalte adyacente a la raíz en la base de la corona; (13) cuerda distal (Dc; distal cord): distan-

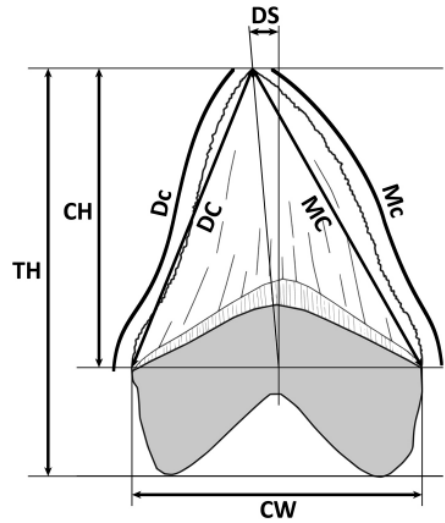

Vista lingual

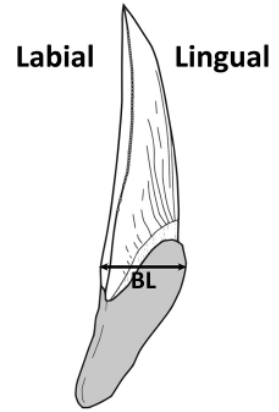

Vista margen distal
Fig. 2. Diferentes mediciones utilizadas en la descripción de los dientes de Carcharocles megalodon. Ver el texto para más información sobre la definición de las medidas y las abreviaciones empleadas. 


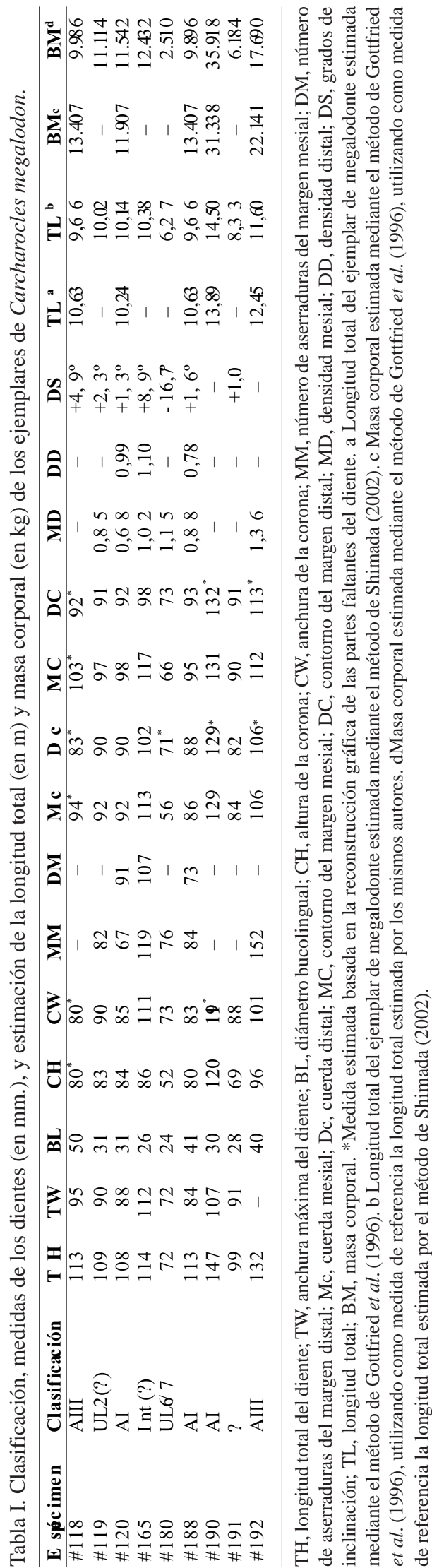

cia entre el ápice de la corona y la extensión distal inferior del esmalte adyacente a la raíz en la base de la corona; (14) grado de inclinación (DS; degree of slant): ángulo comprendido entre una línea dibujada perpendicular a la línea que pasa por las extensiones inferiores de la corona y que pasa por el punto medio de la anchura de la corona (es decir, a mitad de CW), y la línea que emerge del mismo punto y pasa por el ápice de la corona. El ángulo es positivo si la inclinación se dirige hacia el margen distal del diente, y es negativo si está orientado hacia el margen mesial.

Las mediciones TH, TW, BL, $\mathrm{CH}$ y $\mathrm{CW}$ se han tomado mediante un calibre antropométrico. Las mediciones MC y DC se han tomado mediante una cinta métrica flexible no elástica. El recuento de las aserraduras en los márgenes del diente (medidas MM y DM) se tomaron con la ayuda de un estereomicroscopio Leica Wild M8 con una ampliación de $\times 5$, bajo un sistema de iluminación Volpi Intralux 5000. El grado de inclinación (DS) se midió utilizando las imágenes fotográficas de alta resolución de cada uno los especímenes con ayuda del programa Adobe Photoshop CS6 extended. Algunas medidas relacionadas con los dientes \#118, \#180, \#190 y \#192 (Tabla I) se estimaron reconstruyendo gráficamente las partes faltantes de los especímenes.

Estimación de la longitud total y la masa corporal. La carencia de esqueletos fósiles bien preservados de C. megalodon ha forzado a los investigadores a basarse en la morfología del gran tiburón blanco para inferir su aspecto y estimar su tamaño y masa corporal.

La estimación de la longitud total (TL) de los ejemplares de $C$. megalodon se ha realizado basándose en la longitud total del diente (TH) y la altura de la corona $(\mathrm{CH})$, aplicando los métodos de Gottfried et al. (1996) y Shimada (2003). El método de Gottfried et al. (1996) se basa en la relación lineal existente entre la máxima altura del mayor de los dientes anteriores superiores y la longitud total en dicho tiburón. La relación propuesta es la siguiente:

$$
\text { Longitud total }(\mathrm{m} .)=-0,22+0,096 \times[\text { TH de un diente anterior superior }(\mathrm{mm} .)]
$$

Por otra parte, Shimada (2003) propone una regresión basada en la relación entre la altura de la corona y la longitud total, pero teniendo en cuenta todas las posiciones del conjunto de dientes. La relación se calcula de la siguiente manera:

$$
\text { Longitud total }(\mathrm{cm} .)=-2,160+12,103 \times[\mathrm{CH} \text { de cualquier diente }(\mathrm{mm} .)]
$$

Para la estimación de la masa corporal (BM), Gottfried et al. (1996) establecen una relación exponencial ente la longitud total del ejemplar y su masa corporal. La fórmula propuesta es:

$$
\text { Peso }(\text { tonelada })=3,29 \mathrm{E}^{-06} \times\left[\text { TL }(m .)^{3,174}\right]
$$

Cabe aclarar que la unidad 'tonelada' de uso habitual en Estados Unidos (conocida como ton) equivale a 907,18474 kg. De este modo, tras aplicar la fórmula de la estimación de la masa corporal, el resultado se tendrá que por multiplicar por el valor de 907,18474 para obtener el peso estimado en kilogramos según el sistema métrico internacional. 


\section{RESULTADOS}

Los nueve dientes analizados muestran las características diagnósticas típicas de C. megalodon, como son las grandes dimensiones, forma triangular, fino aserramiento de los márgenes de la corona, una cara lingual convexa y una cara labial plana o ligeramente convexa, y un gran cuello en forma de ' $\mathrm{V}$ '; además, los dos lóbulos de la raíz están bien diferenciados y con márgenes basales bien redondeados (Figs. 3, 4 y 5).

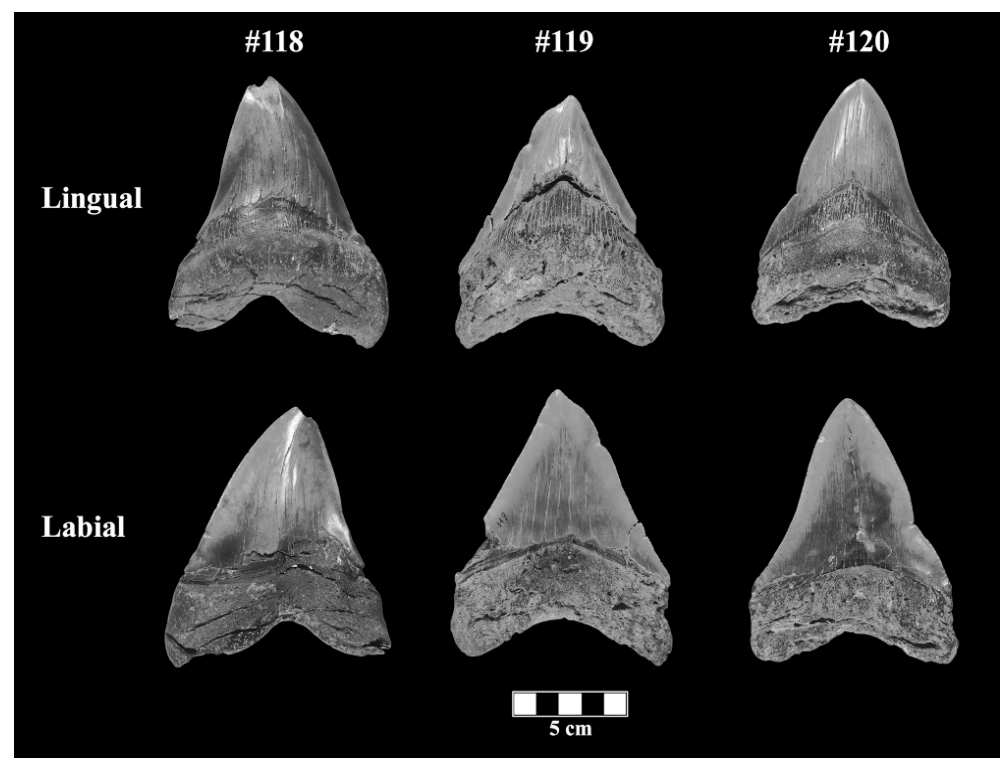

Fig. 3. Vista lingual y labial de los especímenes \#118 (AIII; tercer diente anterior superior), \#119 (UL2(?); probablemente un segundo lateral superior) y \#120 (AI; primer diente anterior superior) de Carcharocles megalodon.

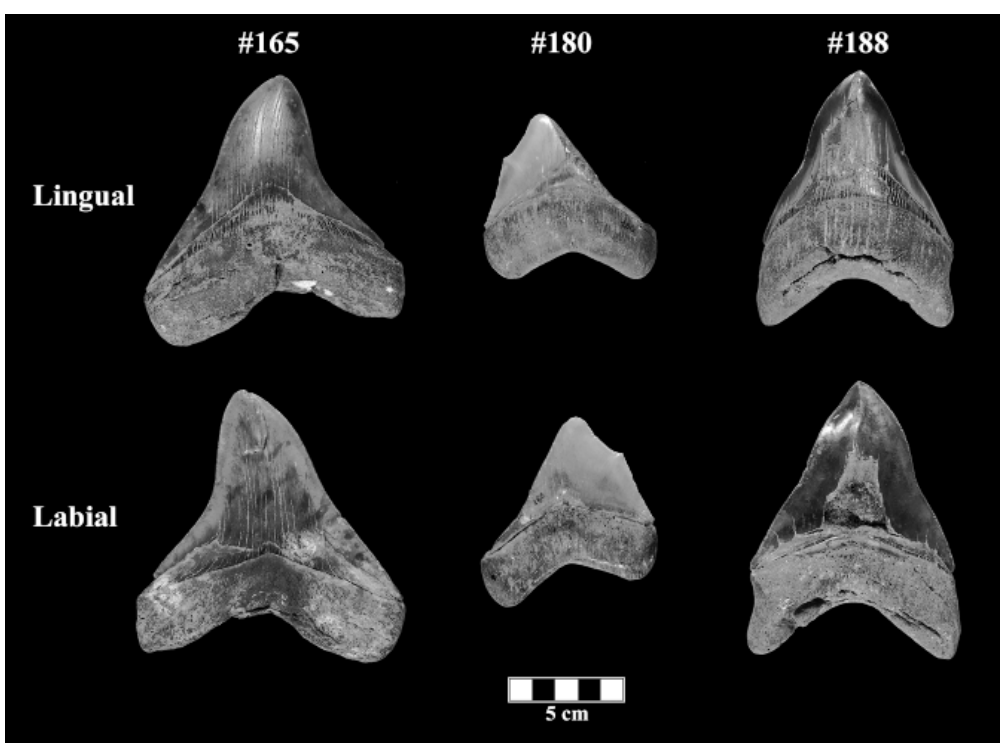

Fig. 4. Vista lingual y labial de los especímenes \#165 (Int(?); probablemente un diente intermedio superior), \#180 (UL6/7; probablemente un sexto o un séptimo diente lateral superior) y \#188 (AI; primer diente anterior superior) de Carcharocles megalodon.
De acuerdo con la anatomía dental, en la arcada superior los dientes anteriores son grandes y tendencialmente simétricos, y aplanados en sentido bucolingual. El diente intermedio es más pequeño respecto a los otros dientes y con el ápice de la corona orientado hacia la sínfisis. La corona de los dientes laterales está generalmente orientada hacia los ángulos mandibulares. Respecto a la arcada inferior, los dientes anteriores tienen una sección redondeada, el diente intermedio está ausente y los dientes laterales son estrechos y están orientados verticalmente (Applegate \& Espinosa-Arrubarrena, 1996; Hubbell, 1996; Mollet et al., 1996). Considerando las características morfológicas expuestas anteriormente y las dimensiones de los dientes, todos los especímenes analizados corresponden a dientes superiores. En particular, los dientes \#120, \#188 y \#190 se pueden clasificar como primeros anteriores superiores (AI). Los dientes \#118 y \#192 presentan las características de los terceros superiores (AIII). La clasificación de los restantes dientes no ha podido ser definida con precisión: el espécimen \#165 podría corresponder a un diente intermedio, el espécimen \#119 probablemente a un segundo lateral (UL2), mientras que el espécimen \#180 correspondería a un sexto o séptimo lateral (UL6/ 7). La identificación del diente \#191 no ha podido ser definida (Figs. 3, 4 y 5). La clasificación y las mediciones de los dientes, así como también la estimación de la longitud total de los ejemplares de Carcharocles megalodon y su masa corporal se muestran en la Tabla I.

Por otro lado, la densidad de las aserraduras de los dientes varía entre 0,85 y 1,36 aserraduras por $\mathrm{cm}$. sobre el margen mesial, y entre 0,78 y 1,10 sobre el margen distal.

La longitud total de los tiburones megalodontes, estimada sobre la base de la altura total de los dientes anteriores, varía entre 10,24-13,89 m. según el método de Gottfried et al. (1996). La masa corporal estimada para estos ejemplares varía entre los 13.407-31.338 $\mathrm{kg}$. La longitud total estimada sobre la base de la altura de la corona de todos los especímenes analizados varía entre 6,27-14,50 m según el método de Shimada (2003). Respecto a la masa corporal, esta varía entre los $2.510-35.918 \mathrm{~kg}$. 


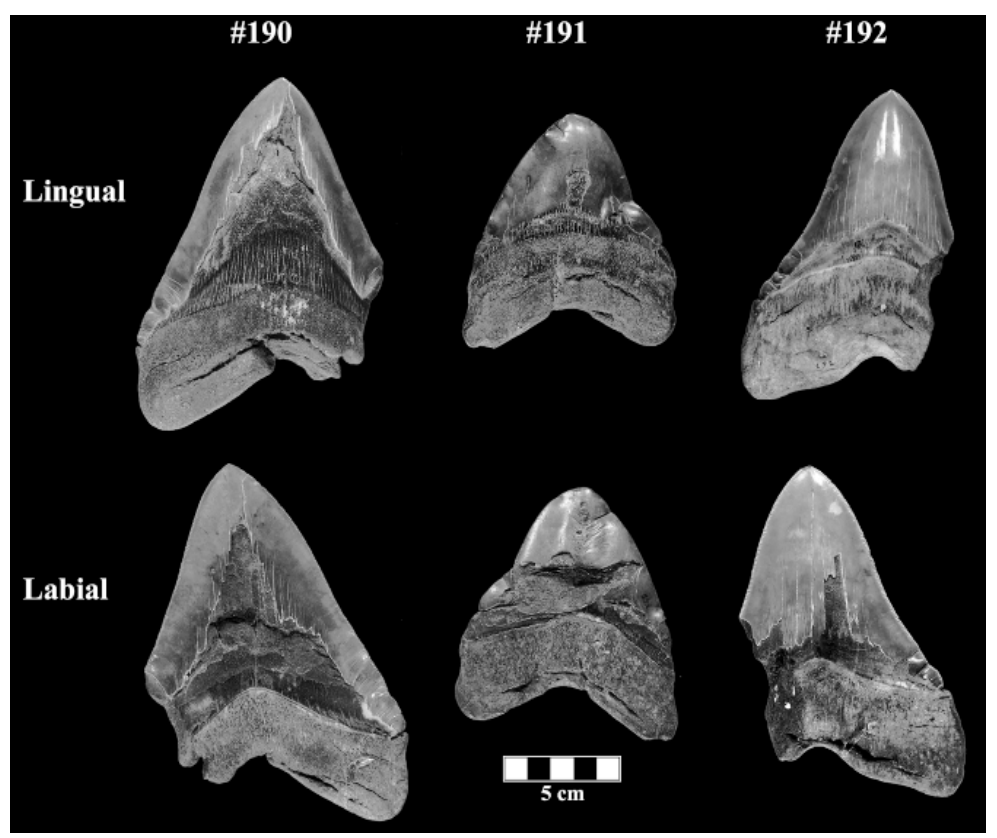

Fig. 5. Vista lingual y labial de los especímenes \#190 (AI; primer diente anterior superior), \#191 (?; diente superior no identificado) y \#192 (AIII; tercer diente anterior superior) de Carcharocles megalodon.

\section{DISCUSIÓN Y CONCLUSIONES}

Carcharocles megalodon es considerado el tiburón más grande y más poderoso que jamás haya existido. Los restos fósiles hallados y la fauna asociada sugieren que esta especie tuvo una distribución cosmopolita en latitudes cálidas/templadas desde el Mioceno medio hasta el Plioceno (15,9-2,6 $\mathrm{Ma})$, encontrándose en lo alto de la cadena alimenticia (Pimiento et al., 2015). En diversas excavaciones se han hallado restos fósiles de cetáceos con profundas marcas de mordedura que coinciden con el tamaño y morfología de los dientes de C. megalodon, lo que indicaría que las ballenas y los delfines eran presas regularmente cazadas por él (Demèrè \& Cerutti, 1982; Cigala-Fulgosi, 1990; Purdy, 1996).

La morfología y el tamaño de los dientes demuestran que $C$. megalodon se alimentaba originariamente de peces y, solo posteriormente, cambió sus hábitos alimenticios prefiriendo los mamíferos marinos que abundaban en los mares templados, es decir, en los mismos ecosistemas en los que vive actualmente el gran tiburón blanco (Carcharodon carcharias) (Demèrè \& Cerutti, 1982; Cigala-Fulgosi, 1990; Applegate \& Espinosa-Arrubarrena, 1996; Purdy, 1996).

Las causas de la extinción de C. megalodon, que tuvo lugar aproximadamente hace 2,6 millones de años, todavía sigue siendo un enigma. No obstante, son cuatro las hipótesis propuestas que aportarían luz a esta extinción (Pimiento et al., 2016): (i) el cambio climático (principalmente el enfriamiento oceánico); (ii) un cambio en la distribución hacia latitudes más altas de las grandes especies de mamíferos marinos que eran presas de C. megalodon; (iii) la disminución en la diversidad de pinnípedos (focas, morsas, leones marinos, etc.) y ballenas que se alimentan por filtración; o (iv) la competencia con grandes ballenas depredadoras (p. ej.: odontocetos, orcas). Las dos primeras hipótesis, que son las más difundidas, implican que $C$. megalodon estaba fisiológicamente restringido a temperaturas más cálidas, ya sea para cazar como para reproducirse. Así, ante el descenso de las temperaturas de los océanos a escala global durante el Plioceno, cetáceos, focas y otros mamíferos marinos de los que se alimentaba $C$. megalodon migraron hacia mares todavía más fríos, donde el tiburón no podía seguirlos y provocaron su extinción. Por otro lado, las dos últimas hipótesis implican que los factores bióticos, como la competencia y la disponibilidad de presas, fueron los principales factores de extinción. Un reciente estudio publicado por Pimiento et al., (2016), apoya estas dos últimas hipótesis. De acuerdo con este estudio, a medida que la diversidad de cetáceos decae, aparecen en escena formidables competidores del megalodonte: ancestros del gran tiburón blanco actual y cachalotes que cazan y se comportan como las orcas de hoy en día. Esta tendencia continúa durante todo el Plioceno, con un número cada vez menor de especies de grandes misticetos y una siempre mayor variedad de depredadores con los que los jóvenes megalodontes tienen que competir por el alimento. En resumen, siempre menos comida a disposición para un número mayor de depredadores. No obstante, el debate sobre la extinción de $C$. megalodon no está cerrado todavía.

Los dientes de mayor tamaño de C. megalodon hallados hasta el día de hoy son un primer superior anterior conservado en el Field Museum of Natural History de Chicago (espécimen PF 1168; TH: 168 mm; TW: 125 mm), y un segundo superior lateral del British Museum of Natural History de Londres (espécimen P10725; TH: 154 mm; TW: 134 mm). Según el método de Gottfried et al. (1996), el espécimen PF 1168 de Chicago pertenecería a un ejemplar de $15,90 \mathrm{~m}$ de longitud, mientras que el espécimen P10725 londinense podría pertenecer a un ejemplar que podría haber alcanzado los 14,56 m. de longitud. La longitud total de los ejemplares de megalodonte correspondientes a los nueve especímenes analizados del Museo Universitario de Chieti (Italia) varía entre un mínimo de 6,27 $\mathrm{m}$ y un máximo de $14,50 \mathrm{~m}$, con un tamaño promedio de $10,06 \pm 2,23 \mathrm{~m}$ y un peso promedio de $13.030 \pm 9.542 \mathrm{~kg}$. Se trata, pues, de valores que se sitúan 
dentro del intervalo de variabilidad del tamaño de esta especie (Pimiento et al., 2015). El espécimen \#190 (primer diente anterior superior; AI) es el diente que tiene un mayor tamaño, el cual pertenecería a un ejemplar con una longitud estimada de 13,89 m (de acuerdo con el método de Gottfried $e t$ al., 1996) o de $14,50 \mathrm{~m}$ (de acuerdo con el método de Shimada, 2003), con un peso estimado entre los 31.338$35.918 \mathrm{~kg}$. Le sigue a continuación el espécimen \#192 (tercer diente anterior superior; AIII), cuya longitud estimada correspondería a un ejemplar de 12,45 m (de acuerdo con el método de Gottfried et al., 1996) o de 11,60 m (de acuerdo con el método de Shimada, 2003), con un peso estimado entre los $17.690-22.141 \mathrm{~kg}$.

Aunque todo lo que se sabe del megalodonte proviene de sus dientes, algunas vértebras y algunas marcas de mordedura halladas, esta información no es suficiente para comprender completamente la compleja biología del animal. Aun así, la filogenia y la paleobiología de la especie $C$. megalodon se reconstruye casi exclusivamente a partir de los dientes, ya que son los únicos restos del animal que se conservan en mayor medida durante el proceso de fosilización. La colección de nueve especímenes dentales en buen estado de conservación del Museo Universitario de Chieti (Italia) implementan el registro fósil ya existente, por lo que su puesta a disposición a la comunidad científica contribuirá a aumentar el conocimiento y completar la información concerniente a los estudios filogenéticos y ecológicos del tiburón megalodonte.

VICIANO, J.; LÓPEZ-LÁZARO, S. \& D'ANASTASIO, R. Fossil teeth of Carcharocles megalodon: The collection of the University Museum of Chieti (Italy). Part I: Morphometric Analysis. Int. J. Morphol., 36(4):1378-1385, 2018.

SUMMARY: The extinct species of shark Carcharocles megalodon (Class: Chondrichthyes; Order: Lamniformes) is considered one of the largest and most powerful predators that populated the temperate seas around the world between the middle Miocene and the Pliocene periods (15.9-2.6 My). The reconstruction of its evolutionary history and ecology is based mainly on the morphological and metric studies of the teeth, which represent the overwhelming majority of the fossil remains of this cartilaginous skeleton species. In this article we present the morphological and metric characteristics of the teeth of Carcharocles megalodon that are housed in the University Museum of Chieti (Italy). In particular, the collection is composed of nine teeth of the upper arch, which belonged to different specimens of variable size and weight (total length range: $6.27-14.50 \mathrm{~m}$; weight range: $2,510-35,918 \mathrm{~kg}$ ). The teeth implement the existing fossil record of Carcharocles megalodon, so that the morphometric data collected will contribute to improve knowledge of the phylogenetic and ecological studies of this species.

KEY WORDS: Megalodon; Fossil tooth; Extinct shark; Lamniformes; Size; Body mass.

\section{REFERENCIAS BIBLIOGRÁFICAS}

Applegate, S. P. \& Espinosa-Arrubarrena, L. The Fossil History of Carcharodon and its Possible Ancestor, Cretolamna: A Study in Tooth Identification. In: Klimley, A. P. \& Ainley, D. G. (Eds.). Great White Sharks: the Biology of Carcharodon Carcharias. San Diego, Academic Press, 1996. pp.19-36.

Applegate, S. P. A status report on the genus Carcharodon, the great white shark, and its fossil record. J. Vertebr. Paleontol., 11(Suppl. 3):14A15A, 1991.

Applegate, S. P. Tooth terminology and variation in sharks with special reference to the sand shark, Carcharias taurus Rafinesque. Contrib. Sci. (Los Angeles Calif.), 86:3-18, 1965.

Bigelow, H. B. \& Schroeder, W. C. Fishes of the Western North Atlantic. Part 1. New Haven, Yale University, 1948.

Capetta, H. Chondrichthyes. II. Mesozoic and Cenozoic Elasmobranchii. Handbook of Paleoichthyology, Vol. 3B. Stuttgart, Gustav Fischer Verlag, 1987.

Casier, E. Note sur la collection des poisons paleochnes et iochnes de l'Enclave de Congo. Ann. Mus. R. Congo Belg., 30:1-28, 1960.

Cigala Fulgosi, F. Predation (or possible scavenging) by a great white shark on an extinct species of bottlenosed dolphin in the Italian Pliocene. Tert. Res., 12(1):17-36, 1990.

Compagno, L. J. V. FAO Species Catalogue. Vol. 4. Sharks of the World. An Annotated and Illustrated Catalogue of Shark Species Known to Date. Part 1 - Hexanchiformes to Lamniformes. Roma, Food and Agriculture Organization of the United Nations, 1984.

D’Anastasio, R.; Cesana, D. T.; Viciano, J.; Sciubba, M.; Nibaruta, P. \& Capasso, L. The possible correlation between dental enamel hypoplasia and a historic natural disaster in the Roman population of Herculaneum (79 AD - central Italy). Anthropol. Anz., 70(4):369-83, 2013.

D’Anastasio, R.; López-Lázaro, S. \& Viciano, J. Fossil teeth of Carcharocles megalodon: the collection of the University Museum of Chieti (Italy). Part II: Paleopathological analysis. Int. J. Morphol., 36(3):841-7, 2018.

Deméré, T. A. \& Cerutti, R. A. A Pliocene shark attack on a cetotheriid whale. J. Paleontol., 56:1480-2, 1982.

Ehret, D. J.; Hubbell, G. \& Macfadden, B. J. Exceptional preservation of the white shark Carcharodon (Lamniformes, Lamnidae) from the early Pliocene of Peru. J. Vertebr. Paleontol., 29(1):1-13, 2009.

Ehret, D. J.; MacFadden, B. J.; Jones, D. S.; Devries, T. J.; Foster, D. A. \& Salas-Gismondi, R. Origin of the white shark Carcharodon (Lamniformes: Lamnidae) based on recalibration of the Upper Neogene Pisco Formation of Peru. Palaeontology, 55(6):1139-53, 2012.

Glickman, L. S. Class Chondrichthyes, Subclass Elasmobranchii. In: Obruchev, D. V. (Ed.). Fundamentals of Paleontology. Vol. 11. Moscow, Nauka, 1964. pp.196-237.

Gottfried, M. D.; Compagno, L. J. V. \& Bowman, S. C. Size and Skeletal Anatomy of the Giant "Megatooth" Shark Carcharodon Megalodon. In: Klimley, A. P. \& Ainley, D. G. (Eds.). Great White Sharks: The Biology of Carcharodon Carcharias. San Diego, Academic Press, 1996. pp.55-66.

Gottfried, M. D.; Compagno, L. J. V. \& Bowman, S. C. Skeletal anatomy of Carcharodon megalodon: inferences based on comparisons with the recent species Carcharodon carcharias. J. Vertebr. Paleontol., 12(Suppl. 3):30A, 1992.

Hubbell, G. Using Tooth Structure to Determine the Evolutionary History of the White Shark. In: Klimley, A. P. \& Ainley, D. G. (Eds.). Great White Sharks: The Biology of Carcharodon carcharias. San Diego, Academic Press, 1996. pp.9-18.

Leriche, M. Les poissons Néogènes de la Belgique. Mem. Mus. R. Hist. Nat. Belg., 32:368-472, 1926.

Mollet, E. F.; Cailliet, G. M.; Klimley, A. P.; Ebert, D. A.; Testi, A. D. \& Compagno, L. J. V. A Review of Length Validation Methods and Protocols to Measure Large White Sharks. In: Klimley, A. P. \& Ainley, 
D. G. (Eds.). Great White Sharks: The Biology of Carcharodon carcharias. San Diego, Academic Press, 1996. pp.91-108.

Nyberg, K. G.; Ciampaglio, C. N. \& Wray, G. A. Tracing the ancestry of the Great White Shark, Carcharondon carcharias, using morphometric analyses of fossil teeth. J. Vertebr. Paleontol., 26(4):806-14, 2006.

Pimiento, C. \& Balk, M. A. Body-size trends of the extinct giant shark Carcharocles megalodon: a deep-time perspective on marine apex predators. Paleobiology, 41(3):479-90, 2015.

Pimiento, C. \& Clements, C. F. When did Carcharocles megalodon become extinct? A new analysis of the fossil record. PLoS One, 9(10):e111086, 2014.

Pimiento, C.; Ehret, D. J.; Macfadden, B. J. \& Hubbell, G. Ancient nursery area for the extinct giant shark megalodon from the Miocene of Panama. PLoS One, 5(5):e10552, 2010.

Pimiento, C.; MacFadden, B. J.; Clements, C. F.; Varela, S.; Jaramillo, C.; Velez-Juarbe, J. \& Silliman, B. R. Geographical distribution patterns of Carcharocles megalodon over time reveal clues about extinction mechanisms. J. Biogeogr., 43(8):1645-55, 2016.

Purdy, R. W. Paleoecology of Fossil White Sharks. In: Klimley, A. P. \& Ainley, D. G. (Eds.). Great White Sharks: The Biology of Carcharodon Carcharias. San Diego, Academic Press, 1996. pp.67-78.

Purdy, R. W.; Schneider V. P.; Applegate, S. P.; McLellan, J. H.; Meyer, R. L. \& Slaughter, B. H. The Neogene Sharks, Rays, and Bony Fishes from Lee Creek Mine, Aurora, North Carolina. In: Ray, C. E. \& Bohaska, D. J. (Eds.). Geology and Paleontology of the Lee Creek Mine, North Carolina, III. Washington D. C., Smithsonian Institution Press, Smithsonian Contributions to Paleobiology, $\mathrm{n}^{\circ}$ 90, 2001. pp.71-202.

Randall, J. E. Size of the great white shark (carcharodon). Science, 181(4095):169-70, 1973

Reolid, M. \& Molina, J. M. Record of Carcharocles megalodon in the Eastern Guadalquivir Basin (Upper Miocene, South Spain). Estud. Geol., 71(2):e032, 2015.

Rothschild, B. M. \& Martin, L. D. Paleopathology: Disease in Fossil Record. London, CRC Press, 1993.

Shimada, K. The relationship between the tooth size and total body length in the white shark, Carcharodon carcharias (Lamniformes: Lamnidae). J. Foss. Res., 35:28-33, 2003.

Uyeno, T.; Sakamoto, O. \& Sekine, H. The description of an almost complete tooth set of Carcharodon megalodon from a Middle Miocene bed in Saitama Prefecture, Japan. Saitama Mus. Nat. Hist. Bull., 7:73-85, 1989.

Viciano, J.; D'Anastasio, R. \& Capasso, L. Odontometric sex estimation on three populations of the Iron Age from Abruzzo region (centralsouthern Italy). Arch. Oral Biol., 60(1):100-15, 2015 b.

Viciano, J.; D'Anastasio, R. \& Capasso, L. Timing of maxillofacial-oral injuries in an individual of the ancient city of Herculaneum (79 AD, Naples, Italy): a case report. Dent. Traumatol., 31(3):215-27, 2015a.

Viciano, J.; López-Lázaro, S.; Cesana, D. T.; D’Anastasio, R. \& Capasso, L. Multiple traumatic dental injuries: A case report in a young individual from the Samnitic necropolis of Opi Val Fondillo (VI-V century BC; Central Italy). J. Archaeol. Sci., 39(2):566-72, 2012.

Ward, D. J. \& Bonavia, C. G. Additions to, and a review of, the Miocene shark and ray fauna of Malta. Cent. Mediterr. Nat., 3(3):131-46, 2001.

\section{Dirección para correspondencia: \\ Ph.D. Joan Viciano \\ Unità Operativa di Antropologia \\ Dipartimento di Medicina e Scienze dell'Invecchiamento \\ Università degli Studi "G. d'Annunzio" di Chieti-Pescara \\ ITALIA}

\author{
E-mail: joan.viciano@unich.it
}

Recibido : 09-02-2018

Aceptado : 20-06-2018 